\title{
Letter to the Editor: Enhanced virtual care in the emergency department as a strategy to safely manage the COVID-19 surge
}

Dear Editor,

Typical workflows in emergency departments (EDs) render recommended coronavirus disease 2019 (COVID-19) physical distancing rules nearly impossible to follow, resulting in thousands of health care workers being infected with COVID-19 to date. ${ }^{1}$ Virtual care may provide a unique opportunity to enable physical distancing in the $\mathrm{ED}$, but it must be done properly to ensure both safety and effectiveness.

Traditional virtual care approaches have often been used in outpatient scenarios where physical exams are not necessary, or otherwise require the assistance of a health care professional to perform a physical exam as part of the assessment. ${ }^{2}$ Adapting this framework to ED patients with suspected COVID-19 introduces challenges, as the most common presenting symptoms for COVID-19 are respiratory in nature and can mimic alternate pathologies (e.g., asthma, congestive heart failure, etc.). A physical examination can, therefore, add important clinical information that will change the management of these patients, but this comes at the cost of potentially exposing providers to COVID-19. Physical examinations also contribute to increased patient satisfaction with the visit, ${ }^{3}$ and can potentially reduce medico-legal concerns that pertain to mis-diagnosis and suboptimal interactions.

To address these challenges and improve adoption of virtual care by both patients and providers, we suggest leveraging tele-equipment to "enhance" the virtual assessment of patients with acute concerns typically seen in ED settings. Historically used for providing care in remote and rural areas, tele-equipment can provide important clinical information that allows providers to more safely and comprehensively assess patients with cardio-respiratory complaints. Several Health Canada-approved devices are currently available, such as electronic stethoscopes, and automated vital signs monitors, that can directly be used by patients to allow providers to remotely examine them. Adopting this "enhanced virtual care" model in the ED has four potential benefits pertaining to the COVID-19 pandemic: (1) increasing safety through limiting physical interaction with patients by physicians and nurses; (2) improving patient satisfaction during the visit and reducing the potential for misdiagnosis; (3) enabling self-isolating, quarantined, or highrisk providers to contribute to ED operations; and (4) reducing personal protective equipment use.

As this pandemic defines a "new normal" for ED processes of care, we anticipate enhanced virtual care to play an increasingly significant role in Canadian EDs. The physical examination continues to be an important element in the assessment of the ED patient, and leveraging technology to enable providers to examine patients remotely will be essential to providing safe and effective care going forward.

\section{Sameer Masood, MD, MPH}

From the Division of Emergency Medicine, Department of Medicine, University of Toronto, and the University Health Network, Toronto, ON

Lucas B. Chartier, MD, MPH (1)

From the Division of Emergency Medicine, Department of Medicine, University of Toronto, and the University Health Network, Toronto, ON

Keywords: Emergency department, COVID-19, virtual care

Competing interests: None declared.

Correspondence to: Dr Sameer Masood, Toronto General Hospital, 200 Elizabeth Street, R. Fraser Elliott Building, Ground Floor, Room 480, Toronto, ON M5G 2C4, Canada; Email: sam1472@mail.harvard.edu

(C) Canadian Association of Emergency Physicians 2020 


\section{REFERENCES}

1. Remuzzi A, Remuzzi G. COVID-19 and Italy: what next? Lancet 2020;395: $1225-1228$.
2. Rosenfield D, Levinter J, Mizzi T, Harvey G. Role for isolated emergency medicine physicians during a pandemic. CFEM 2020. doi: org/10. 1017/cem.2020.390.
3. Kravitz RL, Callahan EJ. Patients' perceptions of omitted examinations and tests: a qualitative analysis. 7 Gen Intern Med 2000;15(1): $38-45$. 